\title{
Enzymatic hydrolysis of apple pulp followed by lactic acid fermentation
}

\author{
Satoshi Noro ${ }^{1}$, Tadashi Takahashi ${ }^{2}$, Jyunji Ichita ${ }^{2}$, Yasuhito Muranaka ${ }^{2}$, and Yoji Kato \\ ${ }^{1}$ Regional Industrial Studies, Department of Regional Society, Graduate School of Hirosaki University, \\ 1 Bunkyo-cho, Hirosaki, Aomori, 036-8560 Japan \\ Fax: 81-0172-39-3436, e-mail: ykato@cc.hirosaki-u.ac.jp \\ ${ }^{2}$ Hirosaki Technical Laboratory, Aomori Industrial Research Center, \\ 80 Fukuro-mach, Hirosaki, Aomori, 036-8363 Japan
}

\begin{abstract}
Apple pomace was fractionated into the water-soluble and water-insoluble materials (apple pulp). The effective saccharification method of apple pulp using PECTINASE HL from Aspergillus sp. (PECase) and CELLULASE "ONOZUKA" 3S (CELase) from Trichoderma viride, was investigated in addition to a screening of favorable microorganism for lactic acid fermentation from Lactobacillus amylophilus (NBRC15881), Rhizopus oryzae (NBRC5384) and Kluyveromyces thermoteleran (AOK A0357-23). On the basis of the results, lactic acid production by $R$. oryzae from the mixture of water-soluble materials of apple pomace and the materials solubilized by hydrolysis of apple pulp with a mixture of PECase and CELase was carried out. Lactic acid was produced at a concentration of $572 \mathrm{~g}$ from $46.8 \mathrm{~kg}$ (wet wt.) of apple pomace at 7 day. The ratio of D- and L-lactic acids was 56:44.

Key words: Apple pomace, Apple pulp, Lactic acid fermentation, Saccharification
\end{abstract}

\section{INTRODUCTION}

Bio-plastic, poly-lactic acid production is one of the major targets for biomass resources. We focused on the residues (apple pulp) of apple fruit extract as the source of lactic acid fermentation. In our previous studies ${ }^{1)}$ on the sugar composition and enzymatic saccharification of apple pulp, we showed that the approximate ratio of carbohydrate in the mono- and oligo-saccharide, watersoluble polysaccharide, pectic substances, hemicellulose, and cellulose fractions of apple pomace was $56: 1: 17: 15$ : 11 , and that about $31 \%$ of apple pulp which corresponds to pectic substances, hemicelulose and cellulose was hydrolyzed to be soluble by PECTINASE HL from Aspergillus sp., and about $9 \%$ was hydrolyzed to be soluble by CELLULASE "ONOZUKA" $3 \mathrm{~S}$ from Trichoderma viride. The present work was conducted to investigate the effective saccharification method with these two enzymes, a screening of microorganism for lactic acid fermentation, and a trial of lactic acid fermentation of the saccharified apple pomace.

\section{MATERIAL AND METHODS}

\section{2-1. Materials}

The apple pomace mainly derived from a cultivar "Fuji" was kindly provided by Gold Nouen, Hirosaki, Aomori. The sample was consisted of water $(78 \%)$, the water soluble mono- and oligo-saccharide $(10 \%)$, the cell-wall polysaccharide $(8 \%)$ and the others $(4 \%)$.

\section{2-2. Enzymes}

PECTINASE HL from Aspergillus sp. (PECase) and CELLULASE "ONOZUKA" 3S (CELase) from Trichoderma viride were purchased from Yakult Pharmaceutical Industry Co. Ltd. and used for saccharification of apple pulp.

\section{2-3. Microorganisms}

Lactobacillus amylophilus (NBRC15881), Rhizopus oryzae (NBRC5384) and Kluyveromyces thermoteleran (AOK A0357-23) were used for the production of lactic acid.

\section{2-4. General methods}

Determination of total carbohydrate and uronic acid, and analyses of the constituent mono- and oligosaccharides in the samples were carried out as reported previously ${ }^{2,3)}$. D/L-Lactic acid was determined by a kit of D/L-lactic acid-UV-method (Roche).

\section{2-5. Stepwise hydrolysis of apple pulp by PECase and CELase}

Apple pomace (wet wt. $400.1 \mathrm{~g}$ ) was mashed and mixed with two volumes of water, and centrifuged to fractionate into the water-soluble materials (mono- and oligo-saccharides and water-soluble polysaccharides) and the water-insoluble materials (apple pulp, wet wt. $222.3 \mathrm{~g}$ ). The apple pulp was mixed with two volumes of water and autoclaved for $20 \mathrm{~min}$ at $121^{\circ} \mathrm{C}$. The autoclaved apple pulp was incubated with PECase (1.1 g) at $45^{\circ} \mathrm{C}$. After $48 \mathrm{hr}$, the materials solubilized by PECase (PEC-M) were removed by centrifugation. The insoluble materials (precipitate) was mixed with two volumes of water, and incubated with CELase $(0.5 \mathrm{~g})$ for $24 \mathrm{hr}$ at $45^{\circ} \mathrm{C}$. The resulting sample was centrifuged to fractionate into the materials solubilized by CELase (CEL-M) and the insoluble materials (final residue, wet wt. $56.1 \mathrm{~g}$ ). Total carbohydrate contents and the sugar compositions of PEC-M and CEL-M were determined respectively.

\section{2-6. Hydrolysis of apple pulp by a mixture of PECase and CELase}

Apple pomace (wet wt. $400.5 \mathrm{~g}$ ) was mashed and mixed with two volumes of water, and centrifuged to fractionate into the water-soluble materials (mono- and oligo-saccharides and water-soluble polysaccharides) and the water-insoluble materials (apple pulp, wet wt. $226.7 \mathrm{~g}$ ). The apple pulp was mixed with two volumes of water and autoclaved for $30 \mathrm{~min}$ at $121^{\circ} \mathrm{C}$. The autoclaved apple pulp was incubated with a mixture of PECase $(1.1 \mathrm{~g})$ and CELase $(1.1 \mathrm{~g})$ for $48 \mathrm{hr}$ at $45^{\circ} \mathrm{C}$. The resulting sample was centrifuged to fractionate into 
the materials (PEC-CEL-M) solubilized by a mixture of PECase and CELase, and the insoluble materials (final residue, wet wt. $50.0 \mathrm{~g}$ ). Total carbohydrate contents and the sugar compositions of PEC-CEL-M were determineed.

\section{2-7. Comparison of L-lactic acid production by} various microorganism.

The $\mathrm{pH}$ of water-soluble materials (WS-M) obtained from apple pomace and the materials solubilized by hydrolysis of apple pulp with a mixture of PECase and CELase (PEC-CEL-M) were adjusted to 6.5 and 5.5 with $\mathrm{NaOH}$ for lactic acid fermentation by $L$. amylophilus and by $K$. thermoteleran and $R$. oryzae, respectively. Then each sample was autoclaved for 20 min at $121^{\circ} \mathrm{C}$. Each microorganism (about $10^{8}$ ) was inoculated into $5 \mathrm{ml}$ of the WS-M (48.6 $\mathrm{mg}$ as Glc equiv.) and into $5 \mathrm{ml}$ of the PEC-CEL-M $(163.7 \mathrm{mg}$ as Glc equiv.). Fermentation was carried out for 7 days at $25^{\circ} \mathrm{C}$. The content of $\mathrm{D} / \mathrm{L}$ - lactic acid, and the total sugar content and sugar composition of each sample were determined.

\section{2-8. Lactic acid production by $R$. oryzae from the} mixture of WS-M and PEC-CEL-M.

Apple pomace (wet wt. $46.8 \mathrm{~kg}$ ) was mashed and mixed with equal volume of water, and the watersoluble materials (WS-M) (yield: 3,546 g as Glc equiv.) were squeezed out by ASAHI PRESS. The water-insoluble materials (apple pulp, wet wt. $39.3 \mathrm{~kg}$ ) was mixed with 2 volumes of water and heated for 30 min at $95^{\circ} \mathrm{C}$. After cooling, the apple pulp was incubated with a mixture of PECase $(197 \mathrm{~g})$ and CELase $(197 \mathrm{~g})$ for $48 \mathrm{hr}$ at $45^{\circ} \mathrm{C}$. The resulting sample was squeezed by ASAHI PRESS to separate the soluble- (PEC-CEL-M, $5,835 \mathrm{~g}$ as Glc equiv.) and insoluble- materials (wet wt. $1.4 \mathrm{~kg}$ ). The WS-M and PEC-CEL-M were combined. The $\mathrm{pH}$ of the combined materials was adjusted to 5.5 with $\mathrm{NaOH}$. $R$. oryzae was inoculated into the combined materials. Fermentation was carried out for 7 days at $25^{\circ} \mathrm{C}$. These procedures are summarized in Fig.1. The content of $\mathrm{D} / \mathrm{L}$-lactic acid during fermentation, and the total sugar content and sugar composition before and after fermentation were determined.

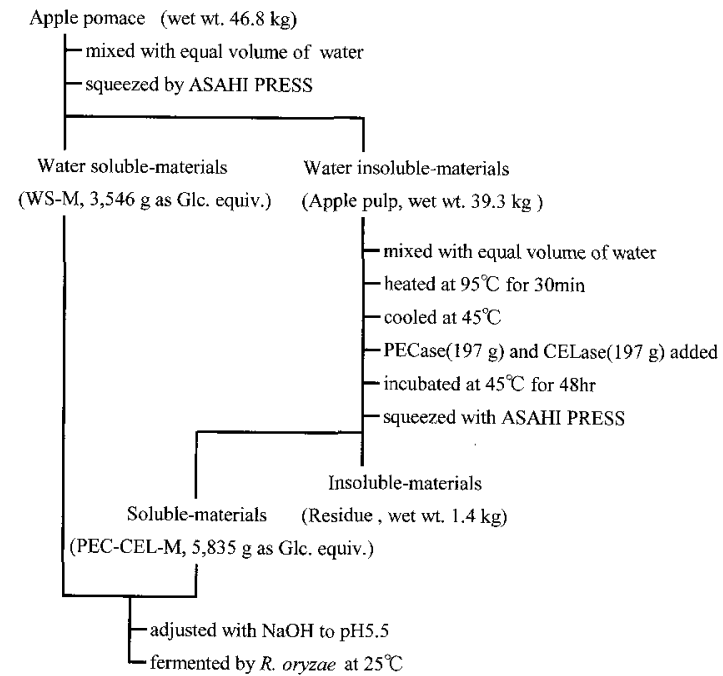

Fig. 1 Flow-chart for the enzymatic hydrolysis of apple pulp followed by lactic acid fermentation.

\section{RESULTS AND DISCUSSION}

3-1. Saccharification of apple pulp.

The influence of different hydrolysis method on the saccharification of apple pulp was examined. Apple pomace was fractionated into the water-soluble (monoand oligo-saccahrides and water-soluble polysaccharides) and the water-insoluble materials (apple pulp). First, the apple pulp $(222.3 \mathrm{~g})$ was treated stepwise with PECase and CELase. Next, the apple pulp (226.7 g) was treated with a mixture of PECase and CELase. Table I shows the yields and sugar composition of the materials solubilized by the enzymatic treatments. There is no distinct deference on the carbohydrate content and sugar composition between them. Considering the cost including water and heating expenses, the treatment of apple pulp by a mixture of PECase and CELase is more efficient than that of apple pulp by stepwise hydrolysis with PECase and CELase.

Table I Yields and sugar composition of the materials solubilized from apple pulp by the enzymatic treatments.

\begin{tabular}{cccccccccc}
\hline $\begin{array}{c}\text { Solubilized } \\
\text { materials }\end{array}$ & $\begin{array}{c}\text { Yield } \\
\text { (g) }\end{array}$ & \multicolumn{6}{c}{ Sugar compositions (\%) } \\
\cline { 3 - 9 } & Fuc. & Ara & Rha. & Gal. & Glc. & Xyl. & Man & U.A. \\
\hline PEC-M & 28.4 & 2.5 & 23.1 & trace. & 16.6 & 42.3 & 10.2 & 0.2 & 5.3 \\
CEL-M & 17.3 & 1.4 & 9.7 & trace. & 8.0 & 67.4 & 10.4 & 0.2 & 2.8 \\
\hdashline PEC-M+CEL-M & 45.7 & 2.1 & 18.0 & trace. & 13.3 & 51.8 & 10.3 & 0.2 & 4.3 \\
\hline PEC-CEL-M & 50.5 & 1.7 & 12.7 & trace. & 9.3 & 58.3 & 10.0 & 0.3 & 7.6 \\
\hline Fuc., Ara., Gal., Glc., Xyl., Man. and U.A. indicate fucose, arabinose,
\end{tabular}
galactose, glucose, mannose and uronic acid, respectively.

\section{3-2. Comparison of L-lactic acid production by} various microorganism.

To examine which microorganism most effectively produce L-lactic acid, WS-M and PEC-CEL-M were fermented for 7 days using L. amylophilus, R. oryzae, and $K$. thermoteleran, respectively. The content of $\mathrm{D} / \mathrm{L}$-lactic acid, and the residual sugar content and sugar composition were determined. Table II shows the content of residual sugar and the sugar composition in 7-days-fermented PEC-CEL-M. Table III shows the yields of D/L-lactic acid of 7-days-fermented WS-M and PEC-CEL-M. The results indicated that $R$. oryzae was effective for the production of L-lactic acid without any other nutrients.

Table II Content of residual sugar and the sugar composition of 7-days-fermented PEC-CEL-M

\begin{tabular}{ccccccccccc}
\hline $\begin{array}{c}\text { Fermentation } \\
\text { with }\end{array}$ & Residual & \multicolumn{6}{c}{ Sugar compositions (\%) } \\
\cline { 3 - 9 }$(\mathrm{mg})$ & Fuc. & Ara. & Rha. & Gal. & Glc. & Xyl. & Man. & U.A. \\
\hline None & 163.7 & 1.7 & 12.7 & trace. & 9.3 & 58.3 & 10.0 & 0.3 & 7.6 \\
A & 129.3 & 1.9 & 11.6 & trace. & 7.8 & 50.3 & 13.1 & 1.0 & 14.2 \\
B & 37.7 & 1.9 & 14.4 & 0.3 & 9.5 & 14.7 & 18.4 & 4.9 & 35.9 \\
C & 62.2 & 2.4 & 14.0 & 4.5 & 10.6 & 21.4 & 18.0 & 4.7 & 24.4 \\
\hline A $:$ L. amylophilus,, B : R. oryzae , C : K. thermoteleran
\end{tabular}

Table III Yields of D/L-lactic acid of 7-days-fermented WS-M and PEC-CEL-M

\begin{tabular}{|c|c|c|c|}
\hline \multirow{2}{*}{ Fermentation with } & Materials & \multicolumn{2}{|c|}{ Yield (mg) } \\
& & L-Lactic acid & D-Lactic acid \\
\hline \multirow{2}{*}{ None } & WS-M & 0.08 & 0.08 \\
\cline { 2 - 4 } & PEC-CEL-M & 0.03 & 0.03 \\
\hline \multirow{2}{*}{ L. amylophilus } & WS-M & 0.94 & 0.10 \\
\cline { 2 - 4 } & PEC-CEL-M & 3.40 & 0.40 \\
\hline \multirow{2}{*}{ R. oryzae } & WS-M & 6.53 & 0.04 \\
\cline { 2 - 4 } & PEC-CEL-M & 9.40 & 0.20 \\
\hline \multirow{2}{*}{ K. thermoteleran } & WS-M & 5.47 & 0.06 \\
\cline { 2 - 4 } & PEC-CEL-M & 1.60 & 0.20 \\
\hline
\end{tabular}


3-3. Lactic acid production by $R$. oryzae from the mixture of WS-M and PEC-CEL-M.

Apple pomace was fractionated into the water-soluble materials (WS-M) and the water-insoluble materials (apple pulp). The apple pulp was hydrolyzed for $48 \mathrm{hr}$ by a mixture of PECase and CELase. Table IV shows the changes in the content of solubilized carbohydrates and their sugar composition during enzymatic hydrolysis of apple pulp. The solubilized carbohydrate increased with the increase in hydrolysis time. Finally $4,658 \mathrm{~g}$ of carbohydrates were solubilized from $39.3 \mathrm{~kg}$ (wet wt.) of apple pulp. The final solubilized carbohydrates (PECCEL-M) consisted of Fuc, Ara, Rha, Gal, Glc, Xyl, Man and UA in the molar ratio of trace:13.4:trace:8.8:62.4: 7.1:trace: 8.3 .

Table IV Changes in the content of solubilized carbohydrates and their sugar composition during enzymatic hydrolysis of apple pulp

\begin{tabular}{cccccccccc}
\hline $\begin{array}{c}\text { Hydrysis } \\
\text { time (hr) }\end{array}$ & $\begin{array}{c}\text { Solubilized } \\
\text { carbohydrate (g) }\end{array}$ & Fuc. & Ara. & Rha. & Gal. & Glc. & Xyl. & Man. & U.A. \\
\hline 0 & 480 & trace. & 0.1 & trace. & 0.3 & 41.3 & 11.7 & trace. & 46.5 \\
1 & 2,409 & trace. & 2.1 & trace. & 2.7 & 55.2 & 8.2 & trace. & 31.8 \\
2 & 2,765 & trace. & 3.5 & trace. & 5.0 & 57.6 & 2.8 & 2.6 & 28.5 \\
3 & 2,944 & trace. & 4.1 & trace. & 5.6 & 57.0 & 2.5 & 3.3 & 27.5 \\
6 & 3,538 & trace. & 9.8 & 0.4 & 7.3 & 64.0 & 4.4 & trace. & 14.1 \\
9 & 3,444 & trace. & 8.6 & 0.9 & 10.0 & 59.0 & 3.7 & trace. & 17.8 \\
24 & 4,010 & trace. & 14.3 & trace. & 9.9 & 60.2 & 5.2 & trace. & 10.4 \\
48 & 4,658 & trace. & 13.4 & trace. & 8.8 & 62.4 & 7.1 & trace. & 8.3 \\
\hline
\end{tabular}

WS-M which corresponds to solbitol, glucose, fructose and sucrose, and the $48 \mathrm{hr}$-hydrolyzate (PEC- CEL-M) were combined, and the combined materials were subjected to lactic acid fermentation by $R$ oryzae. Figure 2 shows the time course of production of lactic acid. In addition, Table $\mathrm{V}$ shows the changes in the content of residual carbohydrates and their sugar composition of a mixture of WS-M and PEC-CEL-M during fermentation by R.oryzae. Lactic acid increased with the increase in fermentation time, and the carbohydrate decreased with the increase in fermentation time. Sugar composition analysis revealed that glucose, fructose and sucrose were mainly converted to lactic acid (Table V). Lactic acid was produced at a concentration of $572 \mathrm{~g}$ from $46.8 \mathrm{~kg}$ (wet wt.) of apple pomace at 7 days (Fig. 2). The ratio of D- and L-lactic acids was $56: 44$. Further studies will be necessary to produce pure L-lactic acid from apple pomace.

Table $V$ Changes in the content of residual carbohydrates and their sugar composition of a mixture of WS-M and PEC-CEL-M during fermentation by R.oryzae.

\begin{tabular}{|c|c|c|c|c|c|c|c|c|c|c|c|c|c|}
\hline \multirow{2}{*}{$\begin{array}{l}\text { Fermentation } \\
\text { days }\end{array}$} & \multirow{2}{*}{$\begin{array}{l}\text { Residual } \\
\text { carbohydrate } \\
\text { (g) }\end{array}$} & \multirow{2}{*}{ (\%) } & \multicolumn{11}{|c|}{ Sugar composition (\%) } \\
\hline & & & Sol. & Fuc. & Ara. & Rha. & Gal. & Glc. & Xyl. & 1. Man. & 1. Frc. & Suc. I & U.A. \\
\hline 0 & 11,016 & 100.0 & 1.7 & trace. & 7.8 & 1.1 & 6.0 & 54.5 & 8.0 & 1.1 & 10.9 & 5.3 & $\overline{5.3}$ \\
\hline$i$ & 4,916 & 44.6 & 4.3 & trace. & 9.8 & 1.8 & 8.9 & 32.4 & 7.2 & 2.4 & 12.3 & 8.91 & 12.0 \\
\hline 3 & 1,532 & 13.9 & 28.5 & trace. & 5.7 & 1.2 & 4.5 & 5.6 & 6.2 & 1.5 & 8.3 & 3.23 & 35.3 \\
\hline 5 & 965 & 8.8 & 39.8 & irace. & 4.6 & 0.7 & 2.6 & 2.1 & 0.5 & 0.6 & 0.2 & 0.64 & 48.3 \\
\hline 7 & 198 & 1.8 & 44.7 & trace. & 1.1 & 0.5 & 0.7 & 0.4 & 0.2 & 0.1 & trace. & trace. 5 & 52.4 \\
\hline
\end{tabular}

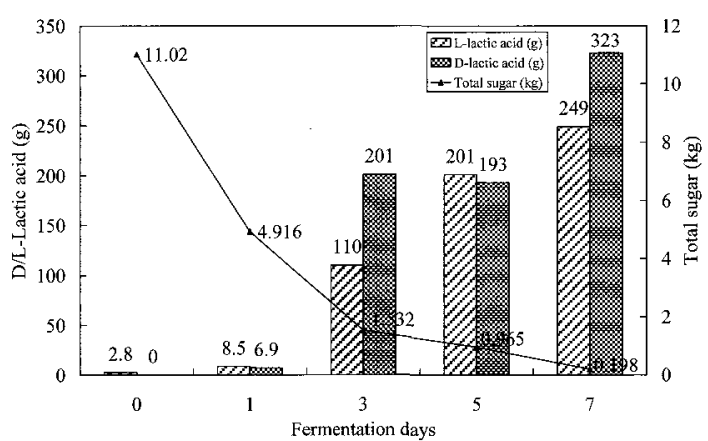

Fig. 2 Production of lactic acid from the mixture of WS-M and PEC-CEL-M by $R$. oryzae.

\section{ACKKNOWLEGEMENTS}

This work was supported by the Department of Ministry of Agriculture, Forestry, and Fisheries of Japan through the National Project of "Biomass Seikatsu Sozo Jigyo Koso".

\section{REFERENCES}

[1] S. Noro, T. Takahashi, J. Ichita, Y. Muranaka and Y. Kato: Sugar composition of apple pulp polysaccharides and their enzymatic hydrolysis. Transactions of the Materials Research Society of Japan, 31(4), 973-976 (2006).

[2] S. Ito, Y. Mitsuishi, T. Okuno and Y. Kato: Changes in the structure of xyloglucan of apple fruit during development. J. Japan. Soc. Hort. Sci., 73(1), 51-56 (2004).

[3] T. Konishi, Y. Mitsuishi and Y. Kato: Analysis of the oligosaccharide units of xyloglucans by digestion with isoprimeverose-producing oligoxyloglucan hydrolase followed by anion-exchange chromatography. Biosci. Biotrchnol. Biochem., 62,2421-2424 (1998).

(Recieved June 6, 2008 ; Accepted September 5, 2008) 